\title{
Optimum Design of FIR Pulse Shaping Filter with Reduced Coefficients
}

\author{
Rupesh Yadav ${ }^{1}$, Puran Gour ${ }^{2}$, Rajesh Nema ${ }^{3}$ \\ ${ }^{I}$ M. Tech Scholar, NIIST, RGTU, Bhopal (M.P.) India; \\ ${ }^{2}$ Professor, Electronics Department, NIIST, RGTU, Bhopal (M.P.) India; \\ ${ }^{3}$ Professor, Electronics Department, NIIST, RGTU, Bhopal (M.P.) India;
}

\begin{abstract}
Pulse shaping filters have been designed and implemented by using Raised cosine filter, Nyquist filter and optimized half band filters for software defined radio (SDR) based wireless applications. In signal processing, a finite impulse response (FIR) filter is a filter whose impulse response (or response to any finite length input) is of finite duration, because it settles to zero in finite time. This is in contrast to infinite impulse response (IIR) filters, which have internal feedback and may continue to respond indefinitely (usually decaying). Filter design is the process of designing a filter, often a linear shift-invariant filter that satisfies a set of requirements, some of which are contradictory. The purpose is to find a realization of the filter that meets each of the requirements to a sufficient degree to make it useful. The filter design process can be described as an optimization problem where each requirement contributes with a term to an error function which should be minimized. Certain parts of the design process can be automated, but normally an experienced electrical engineer is needed to get a good result. The high order demand imposes more hardware requirements, arithmetic operations, area usage, and power consumption when designing and fabricating the filter. Therefore, minimizing or reducing these parameters, is a major goal or target in digital filter design task. In this paper we proposed an Optimum Design of FIR Pulse Shaping Filter with Reduced Coefficients. The performance of the designed filter has been studied in terms of its impulse response as well as magnitude response. For this we take the length parameter as 73 and 85. The results show that the BER performance of the optimized designs is almost identical to the Raised cosine filter with significant reduction in hardware requirements. The result of matlab simulation shows that our approach provides a significant reduction in the number of coefficients without much degradation in its performance.
\end{abstract}

Keywords - FIR, Pulse Shaping Filter, BER, Filter Length, Coefficients

\section{Introduction}

Filters serve as a very useful circuit in many DSP applications. There are quite a few important attributes of FIR filters that have made the use of these circuits to a great extent. For example, it can show linear-phase response over the band of interest if the coefficients of its impulse response are properly chosen [1]. This feature allows the deployment of such filter in practical communication systems. In order to reduce the Inter Symbol Interference (ISI) amongst the symbols in a digital communication system, various FIR filters can be used efficiently as transmitting pulse-shaping filters [2].

IIR filters offer a hardware efficient solution for a given filter characteristic than their FIR counterparts since they can be realized using lower order. Efficient linear phase IIR filter designs based on all pass sub filters have been presented for pulse shaping filters in digital transmission systems [3, 4]. It has been shown that matched transmit-receive filter pairs with very low inter symbol interference (ISI) can be designed using IIR filters [5]. IIR filters employed in mobile communication systems present a hardware design challenge due to the constraints of speed and power consumption.

The RC filters are required to avoid inter-symbol interference and constrain the amount of bandwidth required for transmission [6]. Raised Cosine (RC) is a favorable filter to do pulse shaping as it transition band is shaped like a cosine curve and the response meets the Nyquist Criteria [7]. The first Nyquist criterion states that in order to achieve an ISI-free transmission, the impulse response of the shaping filter should have zero crossings at multiples of the symbol period. A time-domain sinc pulse meets these requirements since its frequency response is a brick wall but this filter is not realizable. We can however approximate it by sampling the impulse response of the ideal continuous filter.

We provide here an overview of FIR Pulse Shaping Filter. The rest of this paper is arranged as follows: 
Section 2 introduces Pulse Shaping Filter; Section 3 describes about recent scenario; Section 4 shows the problem domain. Section 5 shows the Proposed Algorithm; Section 6 shows the simulation result. Section 7 describes Conclusion and outlook.

\section{Pulse Shaping Filter}

Before delving into the details of pulse shaping, it is important to understand that pulses are sent by the transmitter and ultimately detected by the receiver in any data transmission system. At the receiver, the goal is to sample the received signal at an optimal point in the pulse interval to maximize the probability of an accurate binary decision. This implies that the fundamental shapes of the pulses be such that they do not interfere with one another at the optimal sampling point. There are two criteria that ensure non-interference.

Criterion one is that the pulse shape exhibits a zero crossing at the sampling point of all pulse intervals except its own. Otherwise, the residual effect of other pulses will introduce errors into the decision making process. Criterion two is that the shape of the pulses be such that the amplitude decays rapidly outside of the pulse interval.

This is important because any real system will contain timing jitter, which means that the actual sampling point of the receiver will not always be optimal for each and every pulse. So, even if the pulse shape provides a zero crossing at the optimal sampling point of other pulse intervals, timing jitter in the receiver could cause the sampling instant to move, thereby missing the zero crossing point. This, too, introduces error into the decision making process. Thus, the quicker a pulse decays outside of its pulse interval, the less likely it is to allow timing jitter to introduce errors when sampling adjacent pulses.

The rectangular pulse, by definition, meets criterion number one because it is zero at all points outside of the present pulse interval. It clearly cannot cause interference during the sampling time of other pulses. The trouble with the rectangular pulse, however, is that it has significant energy over a fairly large bandwidth.

In fact, because the spectrum of the pulse is given by the familiar $\sin (\pi x) / \pi x$ (sinc) response, its bandwidth actually extends to infinity. The unbounded frequency response of the rectangular pulse renders it unsuitable for modern transmission systems. This is where pulse shaping filters come into play.

If the rectangular pulse is not the best choice for band-limited data transmission, then what pulse shape will limit bandwidth, decay quickly, and provide zero crossings at the pulse sampling times? The raised cosine pulse, which is used in a wide variety of modern data transmission systems. The magnitude spectrum, $P(\omega)$, of the raised cosine pulse is given by:

$$
\mathrm{P}(\omega)=\tau
$$

For $0 \leq \omega \leq \frac{\pi[1-\omega)}{\tau}$

$$
\mathrm{P}(\omega)=\frac{\tau}{2}\left(1-\sin \left(\left(\frac{\pi}{2 \alpha}\right)\left(\omega-\frac{\pi}{\tau}\right)\right)\right)
$$

$$
\text { For } \frac{\pi(1-\alpha)}{\tau} \leq \omega \leq \frac{\pi(1+\infty)}{\tau}
$$

$$
\mathrm{P}(\omega)=0
$$

$$
\text { For } \omega \geq \frac{\pi(1+\infty)}{\tau}
$$

This is also referred to as the impulse response and is given by:

$$
\mathrm{P}(\mathrm{t})=\frac{\left(\operatorname{sinc} \frac{\sin }{\tau}\right)\left(\cos \frac{\operatorname{an\pi } \tau}{\tau}\right)}{1-\left(\frac{2 \sin }{\tau}\right)}
$$

Unlike the rectangular pulse, the raised cosine pulse takes on the shape of a sinc pulse, as indicated by the leftmost term of $p(t)$. Unfortunately, the name "raised cosine" is misleading. It actually refers to the pulse's frequency spectrum, $P(\omega)$, not to its time domain shape, $p(t)$. The precise shape of the raised cosine spectrum is determined by the parameter, $\alpha$, where $0 \leq \alpha \leq 1$. Specifically, $\alpha$ governs the bandwidth occupied by the pulse and the rate at which the tails of the pulse decay. A value of $\alpha=0$ offers the narrowest bandwidth, but the 
slowest rate of decay in the time domain. When $\alpha=1$, the bandwidth is $1 / \tau$, but the time domain tails decay rapidly. It is interesting to note that the $\alpha=1$ case offers a double-sided bandwidth of $2 / \tau$. This exactly matches the bandwidth of the main lobe of a rectangular pulse, but with the added benefit of rapidly decaying timedomain tails. Conversely, Inverse when $\alpha=0$, the bandwidth is reduced to $1 / \tau$, implying a factor-of-two increase in data rate for the same bandwidth occupied by a rectangular pulse. However, this comes at the cost of a much slower rate of decay in the tails of the pulse. The beauty of the pulse-shaping filter concept is that rectangular pulses can be used as the input to the filter. The basic filtering process is synonymous with convolution in the time domain. Also that digital filter provides a convolution operation.

For example, the filter impulse response $h(n)$ is convolved with the input samples to yield the output samples. The convolution of a rectangular pulse (more specifically, a unit impulse) with a raised cosine impulse response results in a raised cosine pulse. The input to the filter is a 1 or 0 (scaled to occupy the full bit width of the filter's input word size) and the output is a raised cosine pulse with all of the time and frequency domain advantages that such a pulse offers.

The ideal raised cosine filter frequency response consists of unity gain at low frequencies, a raised cosine function in the middle, and total attenuation at thigh frequencies. The width of the middle frequencies are defined by the roll off factor constant Alpha, $(0<$ Alpha<=1). In Filter Solutions, the pass band frequency is defined as the $50 \%$ signal attenuation point. The group delay must remain constant at least out to 15 to $20 \mathrm{~dB}$ of attenuation.

When the pass band frequency of a raised cosine filter is set to half the data rate, then the impulse response Nyquist's first criteria is satisfied in that the impulse response is zero for $\mathrm{T}=\mathrm{NTs}$, where $\mathrm{N}$ is an integer, and $\mathrm{T}$ is the data period. Filter Solutions provides analog, IIR and FIR raised cosine filters. FIR are the most accurate and are best to use. However, if it is not possible to use an FIR filter, analog filters may approximate the raised cosine response. The higher the order of the filter, the greater the raised cosine approximation. High order raised cosine filters also produce longer time delays. The lower alpha values use less bandwidth, however, they also produce more ISI due to element value errors and design imperfections. Mathematically, the frequency response may be written as:

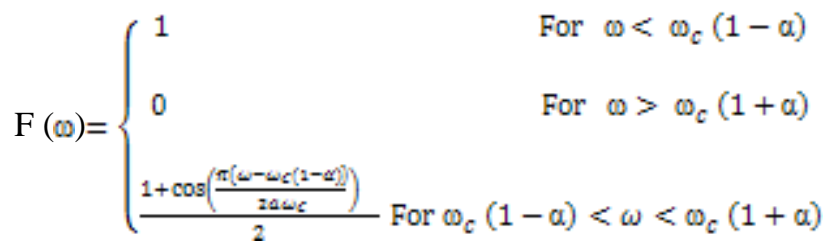

Where $\omega_{c}$ is half the data rate in $\mathrm{r} / \mathrm{s}$

\section{Recent Scenario}

In 2010, Francesco Barale [8] et al. presents the first ultra low power $(5 \mathrm{~mW})$ multi-gigabit pulseshaping filter and clock data recovery circuits fully integrated in a 90-nm CMOS wireless digital radio meeting the specifications of $60 \mathrm{GHz}$ wireless standards. The architecture features a $4.4 \mathrm{Gsps}$ capable 13-tap FIR pulse shaping filter on the TX side, and a dual loop clock data recovery with on chip loop filter on the RX side to suppress the high frequency jitter introduced by the pulse shaping. Using a fully integrated $60 \mathrm{GHz}$ TDD transceiver embedding the presented solution, a $95 \%$ reduction of the high frequency jitter has been measured at the standard nominal $1.728 \mathrm{Gbps}$ data rate. The solution features a minimal power overhead of $5 \mathrm{~mW}$ from $1 \mathrm{~V}$ voltage supply.

In 2010, Mahsa Rezaii et al. [9] propose a novel technique for the design of a convex finite impulse response (FIR) filter. The filter is used to efficiently obtain optimal waveforms that satisfy the spectral mask and maximize the spectrum utilization efficiency in terms of both, the bandwidth, and power allowed by the spectral mask. In addition, it must be noted that the length of the filter is an imperative parameter in our design.

In 2010, S. Chattopadhyay et al. [10] proposed a FIR filter design using Convex optimization method and the performance of the filter has been compared with that obtained by DE technique. Both the Convex optimized as well as DE optimized FIR filter has been used as a transmitting pulse-shaping filter in a Quadrature Phase Shift Keying (QPSK) modulated system to make a comparative study of the system performance. The system performance has also been compared with that of standard Raised Cosine (RC) filter.

In 2011, F. William Mauldin et al. [11] present general filtering method, called the singular value filter (SVF), as a framework for principal component analysis (PCA) based filter design in medical ultrasound 
imaging. The SVF approach operates by projecting the original data onto a new set of bases determined from PCA using singular value decomposition (SVD). The shape of the SVF weighting function, which relates the singular value spectrum of the input data to the filtering coefficients assigned to each basis function, is designed in accordance with a signal model and statistical assumptions regarding the underlying source signals. They applied SVF for the specific application of clutter artifact rejection in diagnostic ultrasound imaging. SVF was compared to a conventional PCA-based filtering technique, which they refer to as the blind source separation (BSS) method, as well as a simple frequency-based finite impulse response (FIR) filter used as a baseline for comparison.

\section{Problem Domain}

The rapidly increasing popularity of mobile radio services has created a series of technological challenges. One of this is the need for power and spectrally efficient modulation schemes to meet the spectral requirements of mobile communications. Linear modulation methods such as QAM, QPSK, have received much attention to their inherent high spectral efficiency However for the efficient amplification of transmitted signal, the Radio Frequency Amplifier is normally operated near the saturation region and therefore exhibit non linear behavior. As a result significant spectral spreading occurs, when a signal with large envelope variations propagates through such an amplifier and creates large envelope fluctuations. Pulse shaping plays a crucial role in spectral shaping in the modern wireless communication to reduce the spectral bandwidth.

Pulse shaping is a spectral processing technique by which fractional out of band power is reduced for low cost, reliable, power and spectrally efficient mobile radio communication systems. It is clear that the pulse shaping filter not only reduces inter symbol interference (ISI), but it also reduces adjacent channel interference. We also want to minimization of the number of coefficients of standard Raised Cosine (RC) pulse-shaping filter of different lengths, without affecting its performance to a great extent. The raised cosine filter is one of the most common pulse-shaping filters in communications systems. In addition, it is used to minimize inter symbol interference (ISI) by attenuating the starting and ending portions of the symbol period.

\section{Proposed Algorithm}

This section presents the details of the proposed algorithm. The main aim of proposed algorithm is to reduce the number of non-zero coefficients used to represent the filter response. For different length considering the value of the roll-off factor to be 0.2 .The algorithm has been depicted by means of a flow chart as follows: 


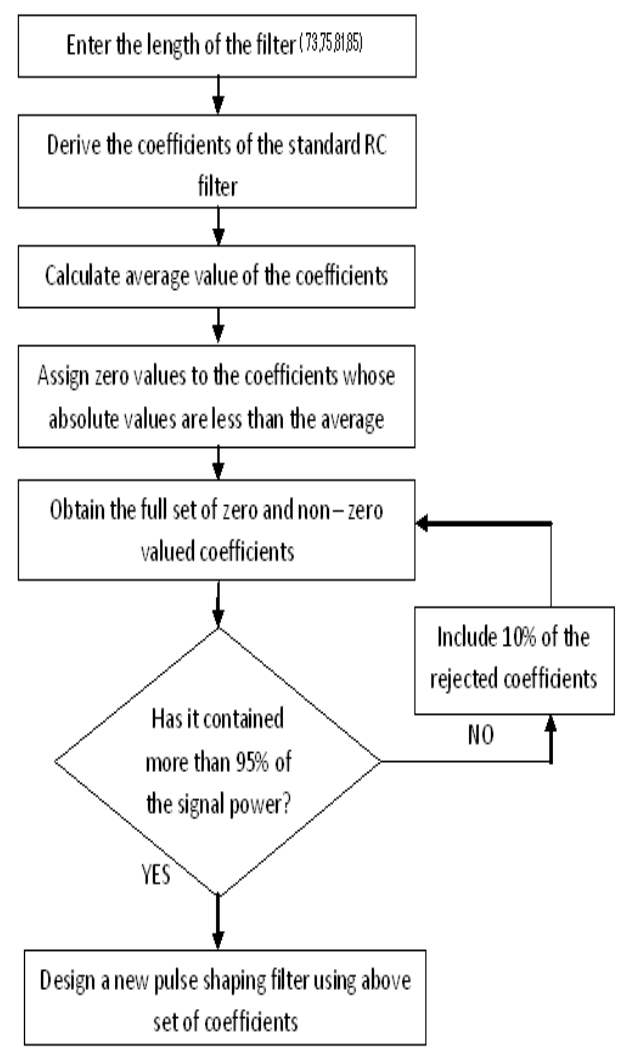

Figure.1. Proposed Algorithm

\section{Results \& Validation}

From the numerical results shown in Table 1, it is clear that the proposed algorithm is capable to design a pulse-shaping filter with significant reduction in the number of non-zero coefficients. More explicitly, considering the length of the pulse-shaping filter to be $73,75,81$ and 85 , the number of non-zero coefficients of the designed pulse-shaping filter has been approximately reduced by $51 \%$ to $59 \%$ respectively.

\begin{tabular}{|c|c|c|c|}
\hline $\begin{array}{c}\text { Length } \\
\text { of the } \\
\text { Filter }\end{array}$ & $\begin{array}{c}\text { Type of } \\
\text { Filter }\end{array}$ & $\begin{array}{c}\text { Number of } \\
\text { Non Zero } \\
\text { Coefficient }\end{array}$ & $\begin{array}{c}\text { Percentage of } \\
\text { coefficients } \\
\text { Reduction }\end{array}$ \\
\hline 73 & $\begin{array}{c}\text { Standard RC } \\
\text { Filter }\end{array}$ & 61 & \multirow{2}{*}{$57 \%$} \\
\cline { 2 - 3 } & $\begin{array}{c}\text { Proposed } \\
\text { Filter }\end{array}$ & 35 & \multirow{2}{*}{57} \\
\hline \multirow{2}{*}{75} & $\begin{array}{c}\text { Standard RC } \\
\text { Filter }\end{array}$ & 63 & \multirow{2}{*}{$59 \%$} \\
\cline { 2 - 3 } & $\begin{array}{c}\text { Proposed } \\
\text { Filter }\end{array}$ & 37 & \multirow{2}{*}{$51 \%$} \\
\hline \multirow{2}{*}{81} & $\begin{array}{c}\text { Standard RC } \\
\text { Filter }\end{array}$ & 71 & \multirow{2}{*}{$51 \%$} \\
\hline
\end{tabular}

www.iosrjournals.org 


\begin{tabular}{|c|c|c|c|}
\hline & $\begin{array}{c}\text { Proposed } \\
\text { Filter }\end{array}$ & 36 & \\
\hline \multirow{3}{*}{85} & $\begin{array}{c}\text { Standard RC } \\
\text { Filter }\end{array}$ & 73 & \multirow{2}{*}{$51 \%$} \\
\cline { 2 - 3 } & $\begin{array}{c}\text { Proposed } \\
\text { Filter }\end{array}$ & 37 & \\
\hline
\end{tabular}

TABLE 1: Proposed Result

To study the role of the proposed filter as a pulse-shaping filter, it has been used in a QPSK-modulated digital communication system and The SNR vs BER plot is shown in Figure 2.

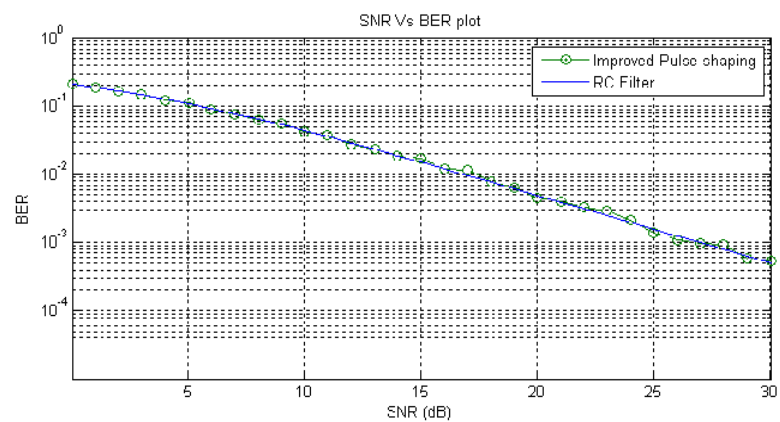

Figure.2. SNR vs BER plot

From the comparison of magnitude response, presented in Figure 3, 4,5 and figure 6, it can be clearly inferred that the performance of the designed FIR pulse-shaping filter is almost similar to that of the standard $\mathrm{RC}$ filter in the pass band region. However, in the stop band region, it is much closer to the standard RC filter. The designed filter produces an attenuation of $60 \mathrm{~dB}$ or more in the stop band which is acceptable. The impulse response shows the reduction of number of non-zero coefficients. With the reduced coefficients the designed filter gives the acceptable performance for practical use.

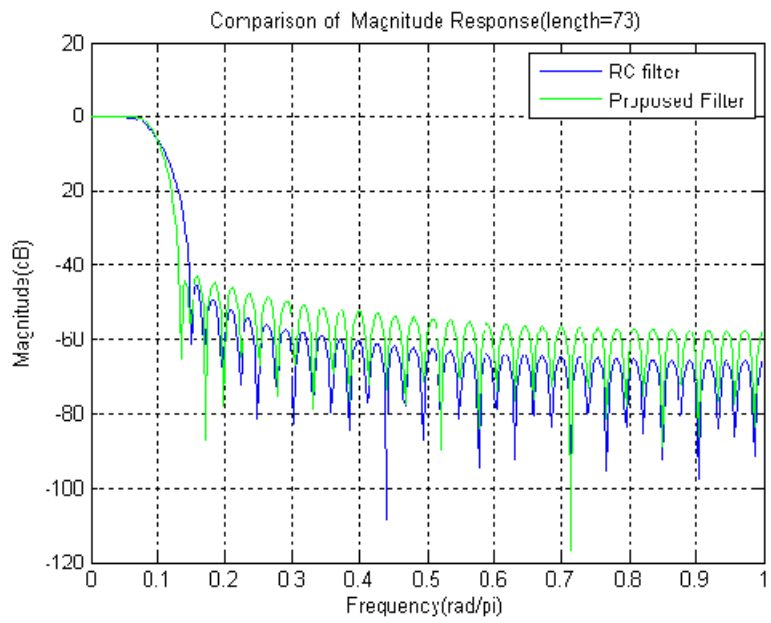

Figure.3.Comparison of Magnitude Response (Length=73) 


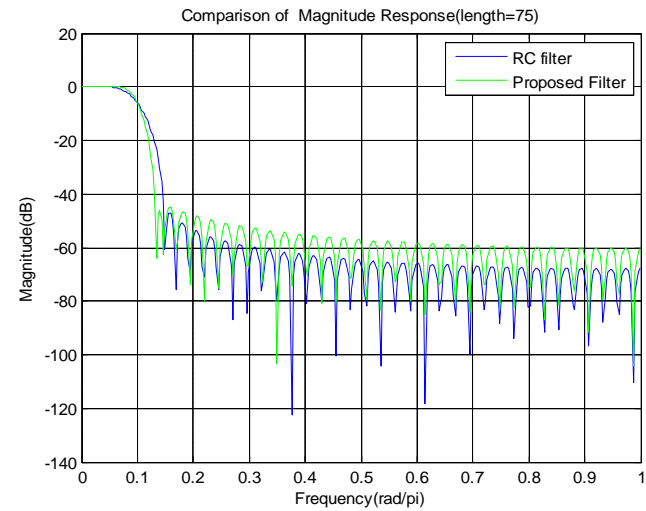

Figure.4.Comparison of Magnitude Response (Length=75)

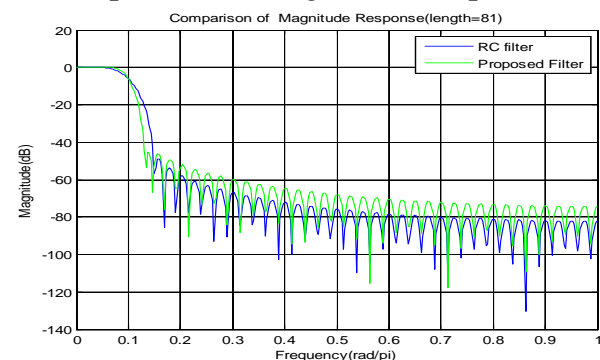

Figure.5.Comparison of Magnitude Response (Length=81)

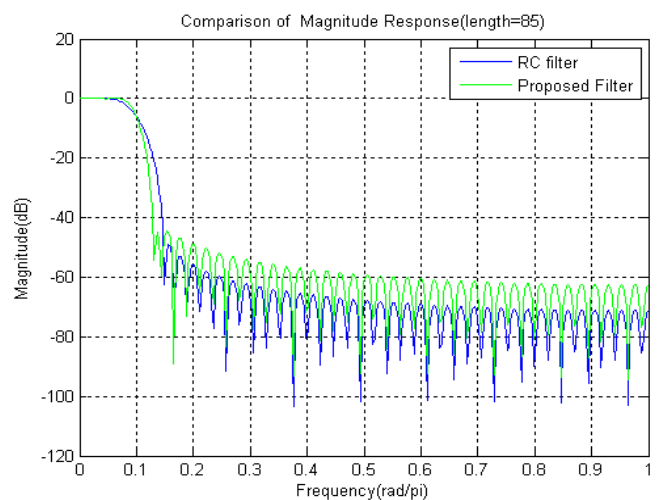

Figure.6.Comparison of Magnitude Response (Length=85)

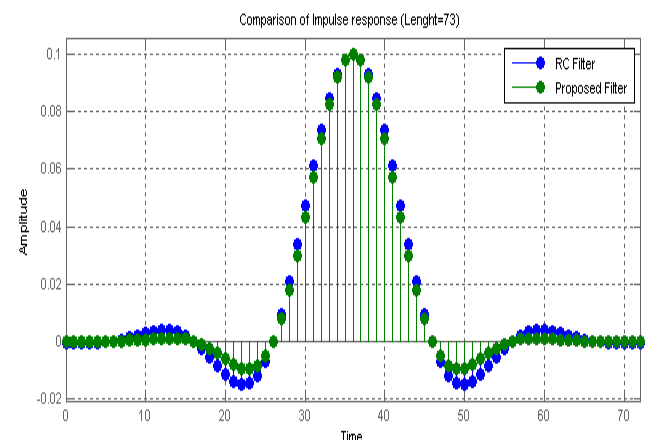

Figure.7.Impulse Response (Length=73) 


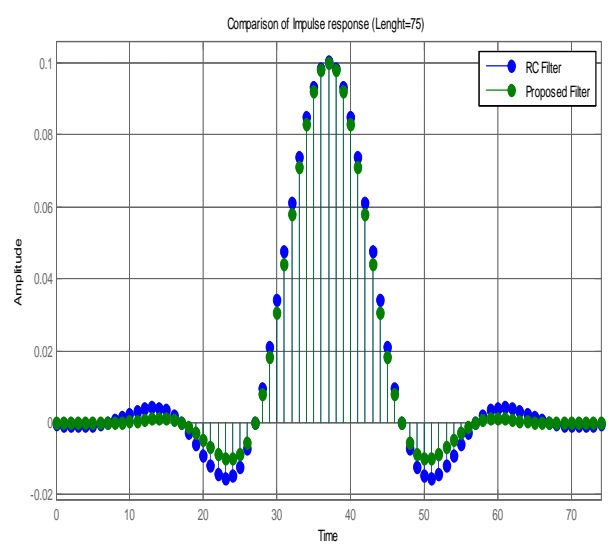

Figure.8.Impulse Response (Length=75)

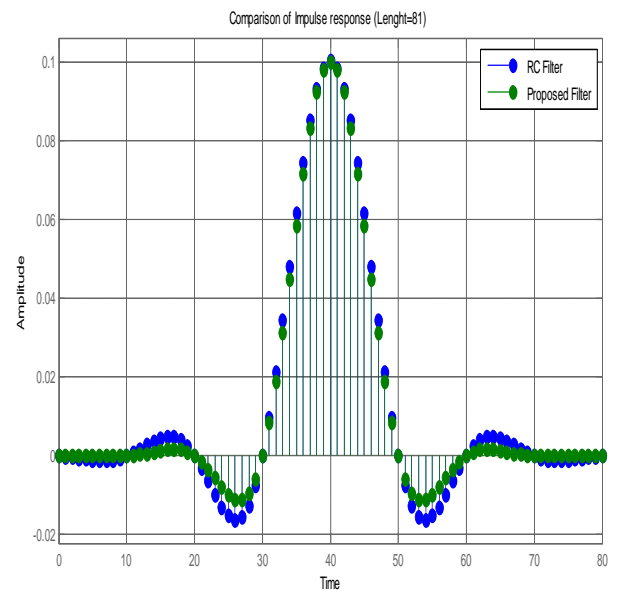

Figure.9.Impulse Response (Length=81)

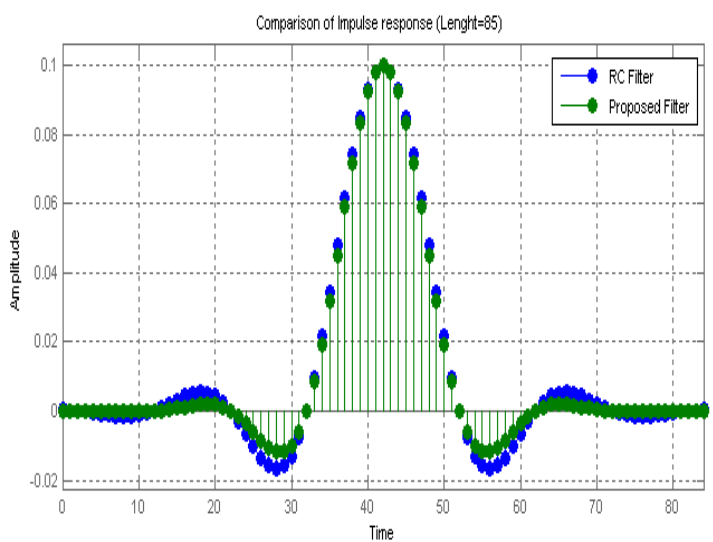

Figure.10.Impulse Response (Length $=85$ )

\section{Conclusion and Outlook}

Finite impulse response (FIR) filters have the advantage of linear phase, guaranteed stability, fewer finite precision errors, and efficient implementation. In contrast, they have a major disadvantage of high order need (more coefficients) than IIR counterpart with comparable performance. The high order demand imposes more hardware requirements, arithmetic operations, area usage, and power consumption when designing and fabricating the filter. Therefore, minimizing or reducing these parameters, is a major goal or target in digital filter design task. High processing speed, low power consumption and low area usage are the major requirements in any digital system. The requirement of high processing speed can be achieved by reducing the computational complexity. For this specific purpose we reduce the coefficient with different length. We also 
compare different BER values with their comparative parameters, which provide more impact on our proposed methodology.

\section{References}

[1] Schilling. R.J. and Harris. S.L., "Fundamentals of Digital Signal Processing using MATLAB", Thomson Learning, India Edition, Delhi,2005, ISBN 813150171X.

[2] Rappaport. T.S, "Wireles Communications: Principles and Practice, $2^{\text {nd }}$ Edition", Chapter 6, Prentice Hall of India Pvt. Ltd., 2007, ISBN 8120323815.

[3] M. Renfors and T. Saramaki, "A class of approximately linear phase digital filters composed of all pass sub filters", in Proc.(IEEE Int. Symp. Circuits Syst.), pp. 678-681, May 1986.

[4] M. Renfors and T. Saramaki, "Pulse-shaping filters for digital transmission systems", in Proc. (IEEE Globecom), vol. 1, pp. 467471, Dec. 1992

[5] M. Renfors and K. Vaisanen, "Efficient IIR filters for pulseshaping and jitter free frequency error detection and timing recovery," in Proc.(IEEE Globecom), vol. 2, pp. 1440-1444, Dec. 1995

[6] K Macpherson, I Stirling, D Garcia, G Rice, Rstewart “Arithmetic Implementation Techniques and Methodologies for 3G Uplink Reception in Xilinx FPGAs" (IEE Conference on 3G Mobile Communication Technologies), pp. 191-195, IEE-2002.

[7] N.M.Zawawi, M.F.Ain, S.I.S.Hassan, M.A.Zakariya, C.Y.Hui and R.Hussin, "Implementing WCDMA Digital Up Converter In FPGA”(IEEE INTERNATIONAL RF AND MICROWAVE CONFERENCE), pp. 91-95, RFM-2008.

[8] Francesco Barale, Gopal B. Iyer, Bevin G. Perumana, Padmanava Sen, Saikat Sarkar, Arun Rachamadugu, Nicolas Dudebout, Stephane Pinel, and Joy Laskar, Pulse Shaping and Clock Data Recovery for Multi -Gigabit Standard Compliant 60 GHz Digital Radio,2010 IEEE.

[9] Mahsa Rezaii," UWB PULSE SHAPING by FIR FILTER to ENHANCE POWER EFFICIENCY",2010 IEEE.

[10] S. Chattopadhyay and S. K. Sanyal," Design of FIR Pulse-Shaping Filter: Superiority of Differential Evolution Optimization over Convex Optimization",(5th European Conference on Circuits and Systems for Communications (ECCSC'10), November 23-25, 2010, Belgrade, Serbia.

[11] F. William Mauldin Jr.*, Dan Lin, and John A. Hossack," The Singular Value Filter: A General Filter Design Strategy for PCABased Signal Separation in Medical Ultrasound Imaging",(IEEE TRANSACTIONS ON MEDICAL IMAGING), VOL. 30, NO. 11, NOVEMBER 2011. 